\title{
A NOTE ON PARACOMPACT SPACES
}

ERNEST MICHAEL ${ }^{1}$

1. Introduction. The purpose of this paper is to prove the following theorem, which asserts that for regular topological spaces paracompactness is equivalent to an apparently weaker property, and derive some of its consequences.

Theorem 1. Let $X$ be a regular topological space. Then $X$ is paracompact if and only if

$\left(^{*}\right)$ every open covering of $X$ has an open refinement $U=U_{i=1}^{\infty} V_{i}$, where each $V_{i}$ is a locally finite collection of open subsets of $X$.

Let us quickly recall the definitions of the terms which are used in the statement of Theorem 1, and which will be used throughout this paper. Let $X$ be a topological space. A collection $R$ of subsets of $X$ is called open (resp. closed) if every element of $R$ is open (resp. closed) in $X$. A covering of $X$ is a collection of subsets of $X$ whose union is $X$; observe that in this paper a covering need not be open. If $R$ is a covering of $X$, then by a refinement of $R$ we mean a covering $V$ of $X$ such that every element of $V$ is a subset of some element of $R$. A collection $R$ of subsets of $X$ is locally finite if every $x \in X$ has a neighborhood which intersects only finitely many elements of $R$. Finally, $X$ is paracompact $[3$, p. 66] if it is Hausdorff, and if every open covering of $X$ has an open, locally finite refinement. (Metric spaces and compact Hausdorff spaces are paracompact (cf. [11] and [3]), and every paracompact space is normal [3].)

In $\$ 2$ we prove Theorem 1, after first obtaining some preliminary lemmas, the first of which may have some independent interest. In $\S \S 2$ and 3 , we derive some of the consequences of Theorem $1 ; \S 2$ deals with the relation of paracompactness to other topological properties, and $\S 3$ deals with subsets and cartesian products of paracompact spaces.

\section{Proof of Theorem 1.}

Lемма $1 .{ }^{2}$ The following three properties of a regular topological space are equivalent.

Received by the editors January 21, 1953.

${ }^{1}$ A. E. C. Fellow.

${ }^{2}$ This result, which will be used in the proof of Theorem 1 below, has other interesting applications. It may, for instance, be used to give a simple proof of the following fact, which seems to be new: Every $T_{1}$-space which is the union of a locally finite collection of closed, paracompact subsets is paracompact. 
(a) $X$ is paracompact (i.e. every open covering of $X$ has an open, locally finite refinement).

(b) Every open covering of $X$ has a locally finite refinement.

(c) Every open covering of $X$ has a closed, locally finite refinement.

Proof. (a) $\rightarrow(\mathrm{b})$ : This is obvious.

(b) $\rightarrow$ (c): Assume (b), and let us prove (c). Let $R$ be an open covering of $X$; we must find a closed, locally finite refinement $\mathcal{A}$ of $R$. Now since $X$ is regular, there exists an open covering $U$ of $X$ such that the collection of closures of elements of $U$ is a refinement of $R$. By assumption, there exists a locally finite refinement $B$ of $V$, and we need only take $\mathcal{A}$ to be the collection of closures of the elements of $\boldsymbol{B}$.

$(c) \rightarrow(a)$ : Assume (c), and let us prove (a). Let $R$ be an open covering of $X$; we must find an open, locally finite refinement $U$ of $R$. Let $A$ be a locally finite refinement of $R$, and let $B$ be a covering of $X$ by open sets, each of which intersects only finitely many elements of $\mathcal{A}$. Now let $\mathscr{W}$ be a closed, locally finite refinement of $\mathcal{B}$. For each $A \in \mathcal{A}$, let $A^{\prime}=X-\cup\{W \in \mathscr{W} \mid A \cap W=\phi\}$; then $A^{\prime}$ is an open set containing $A$, and if $W \in \mathscr{W}$, then $W$ intersects $A^{\prime}$ if and only if $W$ intersects $A$. For each $A \in \mathcal{C}$, pick an $R_{A} \in R$ such that $A \subset R_{A}$. Let $V=\left\{A^{\prime} \cap U_{A} \mid A \in \mathcal{C} A\right.$. Then $U$ is an open refinement of $R$, and since each element of the locally finite covering $\mathscr{W}$ intersects only finitely many elements of $V, V$ is locally finite. This completes the proof.

LEMMA 2. Every countable, open covering $\left\{V_{i}\right\}_{i=1}^{\infty}$ of a topological space has a locally finite refinement $\left\{A_{i}\right\}_{i=1}^{\infty}$ such that $A_{i} \subset V_{i}$ for every $i$.

Proof. Letting $W^{i}=\bigcup_{j=1}^{i} V_{j}(i=1,2, \cdots)$, we need only set $A_{1}=W_{1}$, and $A_{i}=W_{i}-W_{i-1}(i=2,3, \cdots)$.

Proof of Theorem 1 . To prove the nontrivial half of the theorem, let $X$ be a regular space satisfying $\left({ }^{*}\right)$; we must show that $X$ is paracompact. By Lemma 1 , we need only show that every open covering $R$ of $X$ has a locally finite refinement. Now by assumption, $R$ has an open refinement $V=\bigcup_{i=1}^{\infty} V_{i}$, where each $V_{i}$ is locally finite. Let $V_{i}$ be the union of the elements of $V_{i}(i=1,2, \cdots)$; by Lemma 2, there exists a locally finite refinement $\left\{A_{i}\right\}_{i=1}^{\infty}$ of $\left\{V_{i}\right\}_{i=1}^{\infty}$ such that $A_{i} \subset V_{i}$ for all $i$. If we now let $\mathscr{W}=\cup_{i=1}^{\infty}\left\{V \cap A_{i} \mid V \in U_{i}\right\}$, then $\mathscr{W}$ is a locally finite refinement of $R$. This completes the proof.

Let us conclude this section by observing that "regular" cannot be replaced by "Hausdorff" in Theorem 1 (and hence not in Lemma 1, as the proof of Theorem 1 shows). In fact, condition $\left(^{*}\right)$ is clearly satisfied by every second-countable space, and Urysohn $[13, \S 6]$ has given an example of a second-countable Hausdorff space which is not 
regular, and hence certainly not paracompact.

3. Relation of paracompactness to some other topological properties. A topological space is called a Lindelöf space if every open covering of $X$ has a countable subcovering. ${ }^{3}$ Since every Lindelöf space trivially satisfies condition $\left({ }^{*}\right)$, it follows from Theorem 1 that every regular Lindelöf space is paracompact. This result is not new, however, since it was already obtained by Morita [4, Theorem 10].

According to Bing [1, p. 176], a collection $R$ of subsets of a topological space $X$ is called discrete if the closures of the elements of $R$ are disjoint, and if every subcollection of these closures has a closed union. Furthermore, Bing $[1$, p. 176$]$ calls a topological space $X$ strongly screenable if every open covering of $X$ has an open refinement $V=U_{i} V_{i}$, where each $V_{i}$ is a discrete collection of open subsets of $X$. Using Theorem 1, we can now prove the following:

Proposition 1. Let $X$ be a regular topological space. Then $X$ is strongly screenable if and only if $X$ is paracompact.

Proof. If $X$ is strongly screenable, then $X$ certainly satisfies condition $\left(^{*}\right)$, and hence the "only if" part of the proposition follows from our Theorem 1. The "if" part is an immediate consequence of [11, Theorem 2 and Remark after Theorem 1]. This completes the proof.

By a partition of unity on a topological space $X$, we mean a family $\Phi$ of continuous functions from $X$ to the non-negative real numbers such that $\sum_{\phi \in \Phi} \phi(x)=1$ for every $x$ in $X$. A partition of unity $\Phi$ on $X$ is called point-finite if, for every $x$ in $X$, all but finitely many elements of $\Phi$ vanish at $x$; it is called locally finite if every $x$ in $X$ has a neighborhood on which all but finitely many elements of $\Phi$ vanish. If $R$ is a covering of $X$, then a partition of unity $\Phi$ on $X$ is subordinated to $R$ if every element of $\Phi$ vanishes outside some element of $R$ (a function which vanishes nowhere is considered to vanish outside $X$ ). Using Theorem 1, we can now prove the following proposition:

Proposition 2. The following properties of a $T_{1}$-space $X$ are equivalent:

(a) $X$ is paracompact.

(b) Every open covering of $X$ has a locally finite partition of unity subordinated to it.

(c) Every open covering of $X$ has a partition of unity subordinated to it.

${ }^{3}$ As examples of Lindelöf spaces, let us mention second-countable spaces (i.e. spaces with a countable base for the open sets) and $\sigma$-compact spaces (i.e. spaces which are the union of countably many compact subsets). 
Proof. (a) $\rightarrow$ (b): This seems to be known, but we outline the proof for completeness. Let $R$ be an open covering of $X$, and let $\left\{V_{\alpha}\right\}$ be an open, locally finite refinement of $R$. By [3, Theorem 6] there exists a closed covering $\left\{A_{\alpha}\right\}$ of $X$ such that $A_{\alpha} \subset V_{\alpha}$ for all $\alpha$. For each $\alpha$, pick a function $f_{\alpha}$ from $X$ to the closed unit interval such that $f_{\alpha}\left(A_{\alpha}\right)=1$ and $f_{\alpha}\left(X-V_{\alpha}\right)=0$. Let $f(x)=\sum_{\alpha} f_{\alpha}(x)$. If we now define $\phi_{\alpha}(x)=f_{\alpha}(x) / f(x)$ for all $x$, then $\left\{\phi_{\alpha}\right\}$ is a locally finite partition of unity on $X$ which is subordinated to $R$.

(b) $\rightarrow$ (c): This is obvious.

(c) $\rightarrow$ (a): Let $X$ be a $T_{1}$-space which satisfies (c); we must show that $X$ is paracompact. Let us first observe that even a point-finite partition of unity on a space as simple as the unit interval need not be locally finite. To prove that $X$ is paracompact, we will therefore use Theorem 1. It follows easily from (c) that $X$ is completely regular, and we therefore need only verify that $X$ satisfies condition $\left({ }^{*}\right)$. Let $R$ be an open covering of $X$, and let $\Phi$ be a partition of unity on $X$ which is subordinated to $R$. For each positive integer $i$, let $V_{i}$ be the collection of all sets of the form $\{x \in X \mid \phi(x)>1 / i\}$, with $\phi \in \Phi$, and let $U=U_{i=1}^{\infty} V_{i}$. Clearly $V$ is an open refinement of $R$, and we therefore need only check that each $V_{i}$ is locally finite. Let $x_{0} \in X$, and let $i$ be a positive integer; we must find a neighborhood $W$ of $x_{0}$ which intersects only finitely many elements of $V_{i}$. Pick a finite subset $\Phi_{0}$ of $\Phi$ such that $\sum_{\phi \in \Phi_{0}} \phi\left(x_{0}\right)>1-1 / 2 i$, and then pick a neighborhood $W$ of $x_{0}$ such that $\sum_{\phi \in \Phi_{0}} \phi(x)>1-1 / i$ for all $x \in W$. Now $W$ cannot intersect $\{x \in X \mid \phi(x)>1 / i\}$ unless $\phi \in \Phi_{0}$, and therefore $W$ intersects only finitely many elements of $V_{i}$. This completes the proof. ${ }^{4}$

We conclude this section by using Theorem 1 to shed some new light on how metrizability implies paracompactness [11, Corollary 1]. To do this succinctly, let us introduce the following concept: A collection of subsets of a topological space $X$ is called $\sigma$-locally finite if it is the union of countably many locally finite collections of subsets of $X$. Using this terminology, Theorem 1 asserts that a regular space is paracompact if and only if every open covering has an open, $\sigma$-locally finite refinement. On the other hand, Nagata [7, Theorem 1] and Smirnov [8, Theorem 1] have shown that a regular space is metrizable if and only if it has a $\sigma$-locally finite basis for the open sets.

\footnotetext{
4 Using different methods, it is possible to prove the following result, which is more precise than (c) $\rightarrow$ (a) of Proposition 2: Every open covering of a topological space which has a partition of unity subordinated to it, and even every subcovering of such a covering, is normal [12, p. 46], and therefore [11, proof of Theorem 1] has a locally finite refinement.
} 
Thus we see that, for regular spaces, "metric" implies "paracompact" in the same way that "separable metric" implies "Lindelöf." The implications between all these concepts for a regular space are summarized in the following diagram.

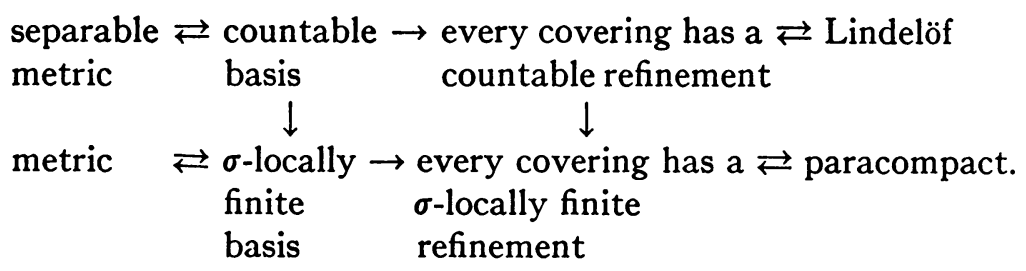

4. Subsets and products of paracompact spaces. In this section we shall use Theorem 1 to prove some results about the paracompactness of subsets and cartesian products of paracompact spaces.

Let us first look at subsets. Certainly an arbitrary subset of a paracompact space need not be paracompact, as can be seen by embedding a non-paracompact, completely regular space in its Stone-Čech compactification. Nevertheless, it was shown by Dieudonné [3] that every closed subset of a paracompact space is paracompact. We now have the following stronger result, which will be used repeatedly in the sequel.

Proposition 3. Every $F_{\sigma}$ subset of a paracompact space is paracompact.

Proof. Let $Y$ be a paracompact space, and let $X$ be an $F_{\sigma}$ subset of $Y$. Since $Y$ is regular, $X$ is regular. By Theorem 1 , we need therefore only show that $X$ satisfies condition $\left({ }^{*}\right)$. Let $R$ be an open covering of $X$; we must find a refinement $U=U_{i=1}^{\infty} V_{i}$ of $R$, where each $V_{i}$ is a locally finite collection of (relatively!) open subsets of $X$. Let $R^{\prime}$ be a collection of open subsets of $Y$ such that $R=\left\{X \cap R^{\prime} \mid R^{\prime} \in R^{\prime}\right\}$. Since $X$ is an $F_{\sigma}$ in $Y, X=\bigcup_{i=1}^{\infty} A_{i}$, where each $A_{i}$ is closed in $Y$. Now for each $i$, let $W_{i}$ be the open covering of $Y$ whose elements are $Y-A_{i}$ and all the elements of $R^{\prime}$. Since $Y$ is paracompact, each $W_{i}$ has an open, locally finite refinement $S_{i}$. Let $\mathcal{T}_{i}$ be the collection of all elements of $S_{i}$ which intersect $A_{i}$; it follows from our construction that $\mathcal{\sigma}_{i}$ is locally finite with respect to $Y$, that $A_{i}$ is contained in the union of the elements of $\mathcal{T}_{i}$, and that every element of $\mathcal{T}_{i}$ is contained in some element of $R^{\prime}$. If we now let $V_{i}=\left\{T \cap X \mid T \in \mathcal{V}_{i}\right\}$, and set $U=U_{i-1}^{\infty} V_{i}$, then all our requirements are seen to be satisfied, and the proof is complete.

REMARK. In the above proof, we have actually demonstrated the following result: If $X$ is an $F_{\sigma}$ subset of a paracompact space $Y$, and if 
$R$ is a relatively open covering of $X$, then $R$ has a relatively open refinement $U$ which is $\sigma$-locally finite with respect to $Y$; i.e. $U$ $=U_{i=1}^{\infty} V_{i}$, where each $V_{i}$ is a locally finite collection of subsets of $Y$.

Let us call a subset $A$ of a topological space a generalized $F_{\sigma}$ if every open set which contains $A$ also contains an $F_{\sigma}$ which contains $A$. Smirnov [9] has proved that every generalized $F_{\sigma}$ (and $a$ fortior $i$ every $F_{\sigma}$ ) subset of a normal space is normal. For paracompact spaces we have the analogous result (formally stronger than Proposition 3 ) that every generalized $F_{\sigma}$ subset of a paracompact space is paracompact; this follows easily from Proposition 3 and the following easily checked fact: If $A$ is a subset of a topological space with the property that every open set which contains $A$ also contains a paracompact set which contains $A$, then $A$ is paracompact. Since Smirnov [9] has shown that every regular Lindelöf space is a generalized $F_{\sigma}$ in its Stone-Cech compactification (which is certainly paracompact), this gives us yet another way of seeing that every regular Lindelöf space is paracompact.

Proposition 3 cannot be strengthened to assert that every completely regular space which is the union of countably many closed, paracompact subsets is paracompact. A striking counter-example is provided by a space (first constructed by J. Dieudonné [4]) which is described in Bourbaki [2, p. 116, Ex. 4]. This space is non-normal, completely regular, and the disjoint union of countably many closed, discrete (hence metrizable, and a fortiori paracompact) subsets; moreover, this space is even locally compact, locally metrizable, has a countable dense subset, and every subset is an $F_{\sigma}$.

Let us now derive some of the consequences of Proposition 3. To begin with, we have C. H. Dowker's result $[5$, p. $634,(\mathrm{~h})]$ that every subset of a paracompact and perfectly normal ${ }^{5}$ space $X$ is paracompact. To prove this, we need only show [3, Theorem $2 b]$ that every open subset of $X$ is paracompact; but this follows immediately from Proposition 3, since every open subset of $X$ is, by definition, an $F_{\sigma}$.

The next application of Proposition 3 deals with cartesian products, to which we now turn our attention. The product of two paracompact spaces need not be paracompact; this was shown by Sorgenfrey [10], who gave an example of a paracompact space $S$ (which is also Lindelöf and perfectly normal) such that $S \times S$ is not

5 A topological space is perfectly normal if it is normal, and if every open subset is an $F_{\sigma}$. (In our terminology, every normal space is Hausdorff.) Every metrizable space is perfectly normal and paracompact. As examples of non-metrizable spaces with both these properties, let us mention the dual of a separable Banach space in the $w^{*}$-topology, and a CW-complex in the sense of J. H. C. Whitehead [14, p. 223]. 
normal. On the other hand, it was shown by Dieudonne [3] that the cartesian product of paracompact space and a compact Hausdorff space is always paracompact. Using Proposition 3, we now generalize this result as follows.

Proposition 4. The cartesian product of a paracompact space and a regular, $\sigma$-compact ${ }^{3}$ space is paracompact.

Proof. Let $X$ be paracompact, and let $Y$ be a regular, $\sigma$-compact space. Then $Y$ is paracompact, hence completely regular, and therefore a subset of a compact Hausdorff space $Z$. (We may, for instance, take $Z$ to be the Stone-Cech compactification of $Y$.) Since $Y$ is $\sigma$-compact, $Y$ is an $F_{\sigma}$ in $Z$, and therefore $X \times Y$ is an $F_{\sigma}$ in $X \times Z$. But $X \times Z$ is paracompact by the above result of Dieudonné, and therefore $X \times Y$ is paracompact by Proposition 3. This completes the proof.

It is not hard to show that Proposition 4 remains true if "paracompact" is everywhere replaced by "regular Lindelöf" or "regular $\sigma$-compact." The proposition cannot be strengthened, however, by replacing " $\sigma$-compact" by "Lindelöf," as is shown by the counterexample of Sorgenfrey which was mentioned earlier.

It seems to be unknown whether the cartesian product of a paracompact space and a metrizable space is always paracompact. But we can prove the following result, using both Theorem 1 and the analogous Nagata-Smirnov characterization of metrizable spaces which was mentioned near the end of Section 3.

Proposition 5. If $X$ is paracompact and perfectly normal, ${ }^{5}$ and if $Y$ is metrizable, then the cartesian product $X \times Y$ is paracompact and perfectly normal.

Proof. According to the above result of Nagata [7] and Smirnov [8], the metric space $Y$ has a $\sigma$-locally finite open basis $U=U_{i=1}^{\infty} V_{i}$, where each $\mho_{i}$ is locally finite. For convenience, we index $U$ by an index set $\mathrm{A}, \mathcal{V}=\left\{V_{\alpha}\right\}_{\alpha \in \mathrm{A}}$, and this notation will be kept throughout the proof.

Let us first show (without using the paracompactness of $X$ ) that every open subset $O$ of $X \times Y$ is an $F_{\sigma}$ in $X \times Y$. For every $\alpha \in \mathrm{A}$ we can clearly find an open $R_{\alpha} \subset X$ such that $R_{\alpha} \times \bar{V}_{\alpha} \subset O$, and such that $\left\{B_{\alpha} \times V_{\alpha}\right\}_{\alpha \in \mathrm{A}}$ is a covering of $O$. Now each $R_{\alpha}=\bigcup_{j=1}^{\infty} B_{\alpha, j}$, where each $B_{\alpha, j}$ is a closed subset of $X$. Let $C_{i, j}=\left\{B_{\alpha, j} \times \bar{V}_{\alpha} \mid V_{\alpha} \in V_{i}\right\}$, and let $C_{i, j}=U C_{i, j}$. Since $C_{i, j}$ is a locally finite collection of closed sets, $C_{i, j}$ is closed. But $O=\bigcup_{i=1, j=1}^{\infty} C_{i, j}$, and hence $O$ is an $F_{\sigma}$.

Let us now prove that $X \times Y$ is paracompact. Since $X \times Y$ is clearly 
regular (as the product of regular spaces), we need only show (by Theorem 1) that every open covering $\mathcal{O}$ of $X \times Y$ has a $\sigma$-locally finite refinement. For each $\alpha \in \mathrm{A}$, pick a collection $\left\{R_{\gamma}\right\}_{\gamma \in \mathrm{r}(\alpha)}$ of open subsets of $X$ such that $\left\{R_{\gamma} \times V_{\alpha}\right\}_{\gamma \in \Gamma(\alpha), \alpha \in \mathrm{A}}$ is an open refinement of $\mathcal{O}$; this can clearly be done. Let $W_{\alpha}=U_{\gamma \in \Gamma(\alpha)} R_{\gamma}$. Then $\left\{R_{\gamma}\right\}_{\gamma \in \Gamma(\alpha)}$ is an open covering of the open (and hence $F_{\sigma}$ ) subset $W_{\alpha}$ of $X$, and hence, by the remark following Proposition 3, it has an open, $\sigma$-locally finite (with respect to $X$ ) refinement $\left\{S_{\beta}\right\}_{\beta \in \mathrm{B}(\alpha)}$. Let $\mathcal{G}=\left\{S_{\beta} \times V_{\alpha}\right\}_{\beta \in \mathrm{B}(\alpha), \alpha \in \mathrm{A}}$; clearly $\mathcal{T}$ is an open refinement of $\mathcal{O}$. But since $\left\{V_{\alpha}\right\}_{\alpha \in \mathrm{A}}$ is a $\sigma$-locally finite collection of subsets of $Y$, and since $\left\{S_{\beta}\right\}_{\beta \in \mathrm{B}(\alpha)}$ is a $\sigma$-locally finite collection of subsets of $X$ for every $\alpha \in \mathrm{A}$, it follows easily that $\mathcal{T}$ is $\sigma$-locally finite, and this completes the proof.

\section{Bibliography}

1. R. H. Bing, Metrization of topological spaces, Canadian Journal of Mathematics vol. 3 (1951) pp. 175-186.

2. N. Bourbaki, Integration, Paris, Hermann, 1952.

3. J. Dieudonné, Une generalization des espaces compacts, J. Math. Pures Appl. vol. 23 (1944) pp. 65-76.

4. - Sur un espace localement compact non metrisable, Anais da Academia Brasileira de Ciencias vol. 19 (1947) pp. 67-69.

5. C. H. Dowker, An embedding theorem for paracompact metric spaces, Duke Math. J. vol. 14 (1947) pp. 639-645.

6. K. Morita, Star-finite coverings and the star-finite property, Mathematica Japonicae vol. 1 (1948) pp. 60-68.

7. J. Nagata, On a necessary and sufficient condition of metrizability, J. Inst. Polytech., Osaka City Univ., Ser. A. Math. vol. 1 (1950) pp. 93-100.

8. Yu. Smirnov, A necessary and sufficient condition for metrizability of a topological space, Doklady Akad. Nauk SSSR. N.S. vol. 77 (1951) pp. 197-200.

9. - On normally disposed sets of normal spaces, Mat. Sbornik N.S. vol. 29 (1951) pp. 173-176.

10. R. H. Sorgenfrey, On the topological product of paracompact spaces, Bull. Amer. Math. Soc. vol. 53 (1947) pp. 631-632.

11. A. H. Stone, Paracompactness and product spaces, Bull. Amer. Math. Soc. vol. 54 (1948) pp. 977-982.

12. J. W. Tukey, Convergence and uniformity in topology, Annals of Mathematics Studies, vol. 2, 1940.

13. P. Urysohn, Über die Mächtigkeit der zusammenhängenden Mengen, Math. Ann. vol. 94 (1925) pp. 262-295.

14. J. H. C. Whitehead, Combinatorial homotopy. I, Bull. Amer. Math. Soc. vol. 55 (1949) pp. 213-246.

The Institute for Advanced Study and

The University of Chicago 